\title{
Internal fields of soot fractal aggregates
}

\author{
Matthew J. Berg ${ }^{1, *}$ and Christopher M. Sorensen ${ }^{2}$ \\ ${ }^{1}$ Department of Physics \& Astronomy, Mississippi State University, 355 Lee Boulevard, Mississippi State, \\ Mississippi 39762, USA \\ ${ }^{2}$ Department of Physics, Kansas State University, Manhattan, Kansas 66506, USA \\ *Corresponding author: matt.berg@msstate.edu
}

Received May 31, 2013; revised August 12, 2013; accepted August 13, 2013;

posted August 13, 2013 (Doc. ID 191530); published September 10, 2013

\begin{abstract}
This work uses the discrete dipole approximation (DDA) to examine the internal electric field within a simulated carbon soot fractal aggregate in fixed and random orientations. For fixed orientations, deviations of the internal field magnitude up to $\pm 50 \%$ from that assumed by the Rayleigh-Debye-Gans Approximation (RDGA) are found. Given the refractive index of the aggregate monomers and conditions for the validity of the approximation, such large deviations are no surprise. Yet despite this deviation, the far-field scattered intensity from such aggregates agrees surprisingly well with that described by the RDGA. Moreover, if the average over an ensemble of many random aggregate-orientations is calculated, both the DDA and RDGA scattered intensities obey the well-known power-law functionality in terms of the scattering wave vector and show a forward-angle intensity-maximum proportional to the square of the number of monomers. The explanation for this lies in the over and under estimations made by the approximation of the internal field, which apparently mostly cancel upon integration to yield the scattered intensity. It is shown that this error cancellation is related to the fractal structure of the aggregate and that the agreement between the DDA and RDGA improves with aggregates of increasing size provided the fractal dimension is less than two. Overall, the analysis suggests that both the special fractal character of the aggregate and its orientational averaging is important to account for the experimentally observed validity of the RDGA despite its poor description of the internal fields. (c) 2013 Optical Society of America
\end{abstract}

OCIS codes: (290.5825) Scattering theory; (290.5850) Scattering, particles; (290.5890) Scattering,

stimulated; (290.1090) Aerosol and cloud effects; (010.1110) Aerosols; (290.5855) Scattering, polarization.

http://dx.doi.org/10.1364/JOSAA.30.001947

\section{INTRODUCTION}

Fractal aggregates are irregularly shaped particle composites that occur via random aggregation of solid particles in aerosols and colloids, e.g., soot aerosols that result from incomplete combustion of hydrocarbon fuels [1]. A substantial amount of work has been devoted to the light-scattering behavior of such aggregates, especially as it relates to the radiative forcing of the atmosphere [1-3]. More broadly, these aggregates are interesting because they offer an opportunity to study scattering from an object with both complex and simple aspects to its morphology. For example, open-flame soot-aggregation yields a dendritic morphology that is highly complicated. Yet despite this complexity, when many aggregates occur simultaneously in random orientations, the observed pattern of scattered light is remarkably simple, following a power-law functionality [Eq. (2) below] in terms of the scattering wave vector $q=2 k \sin (\theta / \overline{2})$, where $k=2 \pi / \lambda$ is the wave number and $\theta$ is the scattering angle.

The popular understanding for why aggregates produce such simple patterns despite their structural complexity originates from the Rayleigh-Debye-Gans Approximation (RDGA) [2]. Central to this approximation is the assumption that electromagnetic interaction between the aggregate's monomers is negligible, i.e., the so-called single-scattering approximation. Moreover, the RDGA assumes that the field within a given monomer is identical to the incident field or, in other words, refraction is neglected. Provided that the aggregate's fractal dimension is $D_{\mathrm{f}}<2$, which is usually the case for soot, this assumption is plausible and has been thoroughly studied [2-4]. Using this RDGA of the internal field, one can derive the experimentally observed power-law functionality of the far-field scattered intensity for aggregates averaged over random orientations [2]. However, certain aggregate characteristics such as the overall size and refractive index lie well beyond the formal range of validity of the RDGA. This recognition has led to many studies concerning the validity of the RDGA, most of which focus on the errors incurred in integrated quantities such as the total scattering and extinction

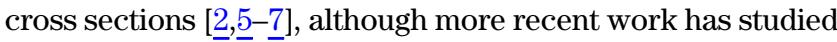
the scattering matrix elements as well [8].

The purpose of this article is not to formally assess the validity of the RDGA for fractal aggregates per se, but to investigate the role played by their special fractal morphology and the orientational averaging. Specifically, this is done from the point of view of the electric field within the aggregate's monomers calculated using the discrete dipole approximation (DDA). The far-field scattered intensity is calculated directly from this field using the Maxwell volume integral equation (VIE). Thus two treatments of an aggregate are compared: the commonly used approximate internal field and scattered intensity as given by the RDGA and the (numerically) exact counterparts given by the DDA. These treatments reveal a surprising behavior. The exact internal field deviates up to $\pm 50 \%$ from the RDGA prediction, yet the scattered intensity agrees very well with that prediction for aggregates in both fixed and random orientations, although the agreement is better in the latter case. The implication is that the over and under estimations of the true internal field made by the RDGA mostly 
cancel upon integration in the VIE to yield little disagreement with the DDA far-field scattered intensity.

\section{AGGREGATE MORPHOLOGY}

Image (a) in Fig. 1 shows a transmission electron microscope (TEM) picture of part of a carbon-soot aggregate captured in an open-burning acetylene flame [9]. This aggregate is composed of roughly spherical carbon monomers. On average, the structure of these aggregates obeys a mass-versus-size scaling law given by Eq. (1) below [2]. Realistic models of aggregate formation have been developed to create simulated aggregates that also obey this scaling law [10]. Image (b) in Fig. 1 shows a simulated aggregate generated by the diffusion-limited cluster aggregation (DLCA) model, which is described, e.g., in [2].

The scaling law for a DLCA fractal aggregates is

$$
N_{\mathrm{m}}=k_{\mathrm{o}}\left(\frac{R_{\mathrm{g}}}{R_{\mathrm{m}}}\right)^{D_{\mathrm{f}}}
$$

where $N_{\mathrm{m}}$ is the number of monomers, $k_{\mathrm{o}}$ is the prefactor, $R_{\mathrm{g}}$ is the aggregate radius of gyration, $R_{\mathrm{m}}$ is the monomer radius, and $D_{\mathrm{f}}$ is the fractal dimension [2]. Aggregates that obey this scaling law possess a fractal-like character in that they show self-similar structure over a range of length scales. The parameters in Eq. (1) related to the aggregate shape are $k_{\mathrm{o}}$ and $D_{\mathrm{f}}$. To get a sense for their influence, [3] shows simulated aggregates for different values of these parameters. For the DLCA aggregate considered here, $k_{\mathrm{o}}$ is roughly constant $k_{\mathrm{o}} \cong 1.3$, and $D_{\mathrm{f}} \cong$ 1.8 [2,11]. Variations of $D_{\mathrm{f}}$ correspond to distinct qualitative changes in the aggregate morphology; $D_{\mathrm{f}}<2$, gives branchlike aggregates that become more compact as $D_{\mathrm{f}}$ increases to $D_{\mathrm{f}}=3$. Aggregates forming from incomplete combustion, like the one in Fig. 1(a), usually correspond to $D_{\mathrm{f}}=1.8$, although examples of multi- $D_{\mathrm{f}}$ aggregates are also known [12-14]. The parameters $R_{\mathrm{g}}, R_{\mathrm{m}}$, and $N_{\mathrm{m}}$ relate to the overall aggregate size, with $R_{\mathrm{g}}$ being the most often used measure. Based upon the TEM image in Fig. 1(a), a nominal monomer size of $R_{\mathrm{m}}=35 \mathrm{~nm}$ is used in this work.

\section{KNOWN SCATTERING BEHAVIOR}

Consider a linearly polarized plane wave $\mathbf{E}^{\text {inc }}$ incident on an aggregate along the positive $z$ axis with polarization along the $x$ axis. The light scattered by the aggregate will be considered in the far field at points in the horizontal scattering plane, i.e., the $y-z$ plane. For common measurements, it is typically the case that many similar aggregates are present simultaneously in random orientations. Then the scattered intensity can be described by the intensity associated with a single aggregate averaged over an ensemble of all possible orientations, although a single orientation also will be considered here. Now, for a $D_{\mathrm{f}}=1.8$ aggregate, the monomers are arranged with approximately two nearest neighbors on average (recall Fig. 1(a) [15]). This means that the monomers are more spread out than the volume elements in a compact, $D_{\mathrm{f}}=3$ particle of the same mass. One could then argue that the electromagnetic coupling between monomers is negligible, and thus, only the influence of the incident wave is important to account for the aggregate's scattering curve. This is the essence of the RDGA; intermonomer coupling is negligible. More specifically, it is possible to show that the RDGA describes the scattered intensity as a Fourier transform of the aggregate's density distribution (see, e.g., [16]). Using this approximation, [2] shows that the scattered intensity for a collection randomly oriented aggregates obeys a power-law functionality in terms of the dimensionless parameter $q R_{\mathrm{g}}$ :

$$
I\left(q R_{\mathrm{g}}\right) \propto\left\{\begin{array}{cc}
N_{\mathrm{m}}^{2} & q R_{\mathrm{g}}<1 \\
N_{\mathrm{m}}^{2}\left(q R_{\mathrm{g}}\right)^{-D_{\mathrm{f}}} & 1 \leq q R_{\mathrm{g}} \leq q R_{\mathrm{m}} . \\
N_{\mathrm{m}}^{2}\left(q R_{\mathrm{g}}\right)^{-4} & q R_{\mathrm{m}}<q R_{\mathrm{g}}
\end{array}\right.
$$

This representation of $I$ in terms of $q$, rather than $\theta$, will be called $Q$-space analysis. Examples of experimental and theoretical studies of $Q$-space analysis to characterize aggregates can be found in $[2,6,9,16,17]$, and these show very good agreement with Eq. (2). For an aggregate in a fixed orientation, the scattering curve will typically contain much more structure than that described in Eq. (2) and will not obey a simple power-law functionality. (a)

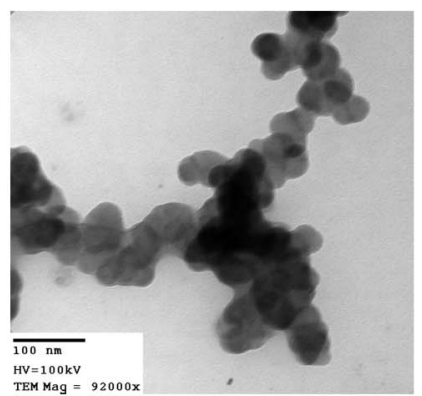

(b)

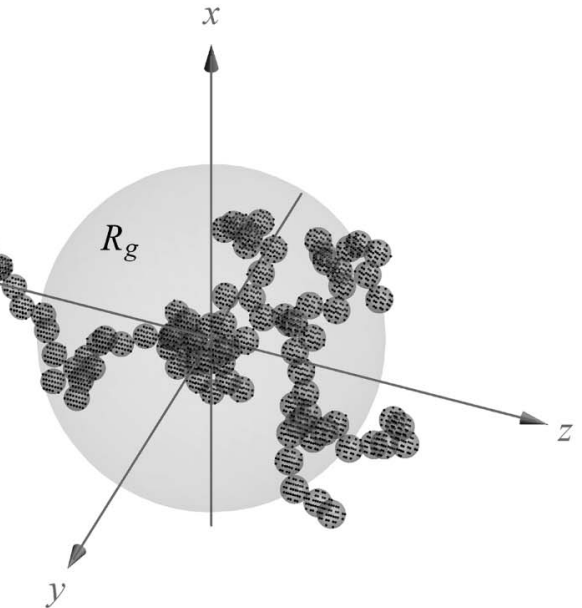

Fig. 1. (a) Open-flame acetylene soot aggregate. (b) DLCA-simulated aggregate. The simulated aggregate contains $N_{\mathrm{m}}=99$ monomers with a fractal dimension of $D_{\mathrm{f}}=1.8$. Also shown in (b) is the DDA lattice residing within the monomers discussed in Section 4 . In this case, the number of lattice sites is $N_{\text {dda }}=3323$ corresponding to approximately 34 sites per monomer. The aggregate's radius of gyration $\bar{R}_{\mathrm{g}}$ is shown by the large gray sphere positioned on the center of mass. 
Upon careful consideration, however, the RDGA's validity becomes quite questionable: Eq. (1) for $D_{\mathrm{f}}=1.8$ soot shows that an aggregate's density decreases with increasing aggregate size; thus one could plausibly argue that intermonomer coupling is negligible. Yet the refractive index of the soot material is relatively strong, estimated to be $m=1.57+0.56 i$ at $\lambda=532 \mathrm{~nm}[2,18]$. Now, in the context of the aggregate monomers, the formal condition for the validity of the RDGA is that both $|m-1| \ll 1$ and $k R_{\mathrm{m}}|m-1| \ll 1$, which given the typical range of $k R_{\mathrm{m}} \cong 0.1-0.5$ for soot, these conditions are not well satisfied [19]. Moreover, it not obvious why contingent monomers with such high refractive index should be treated as independent scatterers. Thus a careful examination of the aggregate's internal field and scattering behavior is warranted.

\section{INTERNAL FIELDS}

To study an aggregate's internal field requires a versatile numerical model that can accurately handle the complex geometry with the strong refractive index. While the T-matrix method is perhaps the most appropriate choice for this application given its analytical capabilities, its current implementations do not readily provide access to the internal field; that information is encoded in the far-field scattered wave via the boundary conditions at each monomer's surface. The DDA, however, formulates the scattered wave directly in terms of the internal field [see Eq. (ㅁ) below] and thus provides a natural choice for this study.

The DDA is applied to a DLCA-simulated aggregate that displays a single fractal dimension indicative of soot, $D_{\mathrm{f}}=1.8$. To do this, the aggregate is superimposed on a large cubic lattice of dipole moments $\mathbf{p}$, where the dipoles residing outside of the aggregate's monomers are set to zero. The fineness of the lattice determines how well the aggregate shape is represented and is quantified by the number of dipoles per monomer $N_{\text {dda }} / N_{\mathrm{m}}$ where $N_{\text {dda }}$ is the number of dipoles residing in the entire aggregate. Figure 1(b) shows the simulated aggregate superimposed on the lattice. In this example, $N_{\text {dda }} / N_{\text {m }} \cong 34$, which one can see adequately represents the aggregate structure, although fine details such as monomer-monomer "necking" are not well represented. To model many aggregates in random orientations, a total of $N_{\text {ori }}$ random directions are selected uniformly, onto which the propagation direction of the incident wave is rotated. The internal or scattered field quantities are then calculated for the same aggregate in each of these orientations. Finally, the ensemble average of the quantities is calculated following Section 3.2 in [20].

Several techniques are used to study the magnitude and direction of the monomer internal field $\mathbf{E}^{\text {int }}$ and to compare these to the RDGA. At each lattice site $\mathbf{r}_{i}$ in the aggregate, the relative, orientationally averaged deviation in magnitude $\delta_{i}$ between the RDGA field and the true (at least within the accuracy of the DDA) internal field, which is proportional to the site's dipole moment, is calculated via

$$
\delta_{i}=\frac{1}{N_{\text {ori }}} \sum_{n=1}^{N_{\text {ori }}} \frac{\left|\mathbf{E}_{n}^{\text {int }}\left(\mathbf{r}_{i}\right)\right|-\left|\mathbf{E}_{n}^{\text {inc }}\left(\mathbf{r}_{i}\right)\right|}{\left|\mathbf{E}_{n}^{\text {inc }}\left(\mathbf{r}_{i}\right)\right|} .
$$

If $\delta_{i}=0$, then the orientationally averaged field at site $\mathbf{r}_{i}$ is equivalent in magnitude to what the RDGA predicts, whereas $\delta_{i} \neq 0$ indicates \pm percent deviations from the RDGA. To study the directional character of the field, each lattice site is assigned a unit vector representing the field's direction at that site. Each aggregate orientation results in a different unit vector, all of which are then averaged to yield a single vector $\mathbf{e}_{i}$ as

$$
\mathbf{e}_{i}=\frac{1}{N_{\text {ori }}} \sum_{n=1}^{N_{\text {ori }}} \frac{\operatorname{Re}\left\{\mathbf{E}_{n}^{\text {int }}\left(\mathbf{r}_{i}\right)\right\}}{\left|\operatorname{Re}\left\{\mathbf{E}_{n}^{\text {int }}\left(\mathbf{r}_{i}\right)\right\}\right|} .
$$

To visualize these directions, a point is plotted on the surface of the unit sphere corresponding to the direction given by the $\mathbf{e}_{i}$ vector. Since $\mathbf{E}^{\text {inc }}$ is polarized along the $x$ axis, these points would be coincident with the $x$-axis poles of the unit sphere if the RDGA holds. A dispersion of points away from these poles indicates a departure in field direction from the RDGA.

Now consider the application of Eqs. (3) and (4) to the aggregate in Fig. 1(b). The number of monomers is $N_{\mathrm{m}}=99$ and the total number of lattice sites is $N_{\mathrm{dda}}=3323$. This gives an average of 34 dipoles per monomer; a slight variation in this number from monomer to monomer is due to lattice-shape errors (see [21]). The size parameter of the aggregate is $k R_{\mathrm{g}}=5.4$, the monomer size parameter is $k R_{\mathrm{m}}=0.42$, and the refractive index is $m=1.57+0.56 i$. These values are representative of carbon soot aggregates illuminated by visible light [2,18]; for example, if $\lambda=532 \mathrm{~nm}, R_{\mathrm{g}}=457 \mathrm{~nm}$, and $R_{\mathrm{m}}=35 \mathrm{~nm}$. One can compare these values to Fig. 1(a) to see satisfactory agreement with actual soot.

Figure $\underline{2}$ shows the aggregate in a single orientation with each monomer assigned a color given by

$$
\Delta_{i}=\frac{1}{V_{\mathrm{m}}} \int_{V_{\mathrm{m}}} \frac{\left|\mathbf{E}^{\mathrm{int}}\left(\mathbf{r}_{i}\right)\right|-\left|\mathbf{E}^{\mathrm{inc}}\left(\mathbf{r}_{i}\right)\right|}{\left|\mathbf{E}^{\text {inc }}\left(\mathbf{r}_{i}\right)\right|} \mathrm{d} V,
$$

where $V_{\mathrm{m}}$ is the monomer volume. The quantity $\Delta_{i}$ represents the fractional (expressed as percent in Fig. 2 ) deviation in magnitude of the internal field throughout a monomer from that predicted by the RDGA. Note that this is not an orientationally averaged quantity unlike much of the following analysis. One can clearly see from Fig. 2 that some monomers

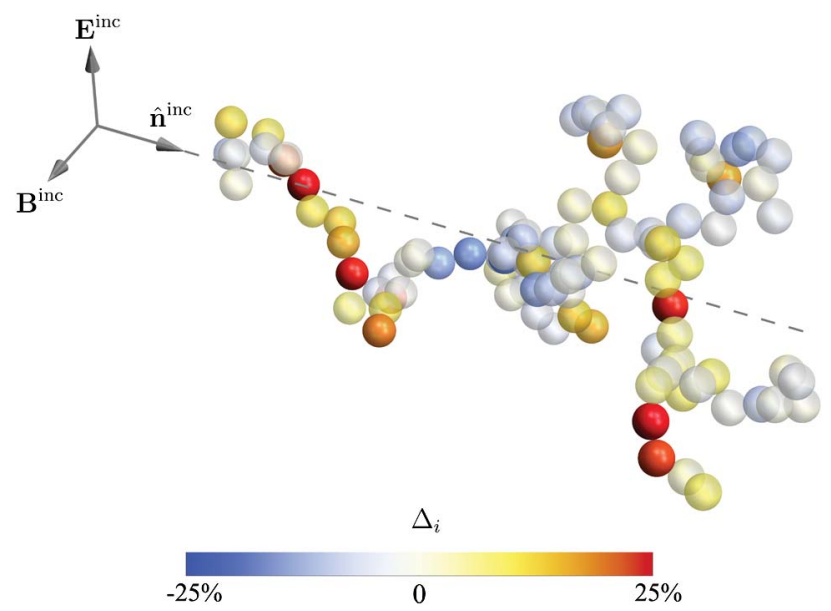

Fig. 2. Percent deviation of the monomer internal field from the RDGA. Shown here is the DLCA-simulated aggregate in Fig. 1(b) with each monomer color-coded according to Eq. (5). 
have $\pm 25 \%$ deviation from the RDGA expectation, and most are at least $\pm 10 \%$ from this expectation.

Given the significant deviations of the internal field seen in Fig. 2, one might expect that the RDGA would be equally poor in describing the far-field scattered intensity $I$. Using the DDA, accurate scattered intensity distributions can be calculated via the far-field scattering amplitude $\mathbf{E}_{1}^{\text {sca }}$, which is essentially a discretized form of the VIE [21]:

$$
\mathbf{E}_{1}^{\mathrm{sca}}(\hat{\mathbf{r}})=\frac{k^{2}}{4 \pi \varepsilon_{\mathrm{o}}}(\stackrel{\leftrightarrow}{\mathbf{I}}-\hat{\mathbf{r}} \otimes \hat{\mathbf{r}}) \cdot \sum_{i=1}^{N_{\mathrm{dda}}} \mathbf{p}_{i} \exp \left(-i k \hat{\mathbf{r}} \cdot \mathbf{r}_{i}\right)
$$

where $\stackrel{\leftrightarrow}{\mathbf{I}}$ is the $3 \times 3$ identity dyadic, $\hat{\mathbf{r}}$ is the direction to the observation point, and $\hat{\mathbf{r}} \otimes \hat{\mathbf{r}}$ is the dyadic formed by the direct product of $\hat{\mathbf{r}}$ with itself. Equation (6) describes the scattered wave amplitude as a Fourier transform of the aggregate's internal field as represented by the dipole moments $\mathbf{p}_{i}$ associated with each volume element in the aggregate. In the RDGA, this internal field is replaced by the incident field, in which case Eq. (6) can be simplified to a form that describes the scattered wave as the Fourier transform of the aggregate's spatial structure (see [22]). Equipped now with the DDA and RDGA far-field scattered intensity, a comparison between the two can be done, and this is shown in Fig. 3. Referring to plot (a), the comparison is done for the aggregate in a single orientation and averaged over $N_{\text {ori }}=500$ orientations. For the single orientation, the RDGA scattered intensity follows the DDA intensity surprisingly well. The agreement is improved when orientational averaging is done. To explore the consequences of this averaging in more detail, plot (b) presents the DDA scattered-intensity for the aggregate averaged over a variety of random orientations. One can see that only after roughly 500 orientations are included does the scattered intensity take on the power-law functionality of Eq. (2). Thus, Figs. 2 and 3 give a conflicting message for the validity of the RDGA. Regarding the internal field, the RDGA only poorly represents the true internal field. The far-field scattered intensity, however, is quite well described by the RDGA. Moreover, the success of the RDGA for the scattered intensity appears to only weakly depend on the average over many aggregateorientations.

Because the DDA calculations do not analytically average the scattered intensity, one must establish if enough orientations are included such that the ensemble average converges to its true value. The convergence is established from the evolution of the $Q$ space scattering curves, which is presented in Fig. $3(\mathrm{~b})$. One can see from these plots that the average converges well for $N_{\text {ori }}=500$ and agrees with Eq. (2).

To further compare the internal field and scattered intensity, Fig. 4 shows the field-direction points $\mathbf{e}_{i}$ of Eq. (4) in row (a), the relative magnitude-deviation $\delta_{i}$ of Eq. (3) in row (b), and the polarization state of the far-field scattered wave in row (c). Each column shows these quantities for $N_{\text {ori }}=1,10$, and 500 aggregate orientations. One can see in row (a) that most lattice sites in the aggregate have field directions close to the $x$-axis poles, consistent with the RDGA. Interestingly, as more orientations are included in the ensemble, the dispersion of points appears to narrow only slightly, unlike the narrowing seen in the magnitude-deviation histograms in row (b). Moreover, the shape of the distributions in row (b) shows that the
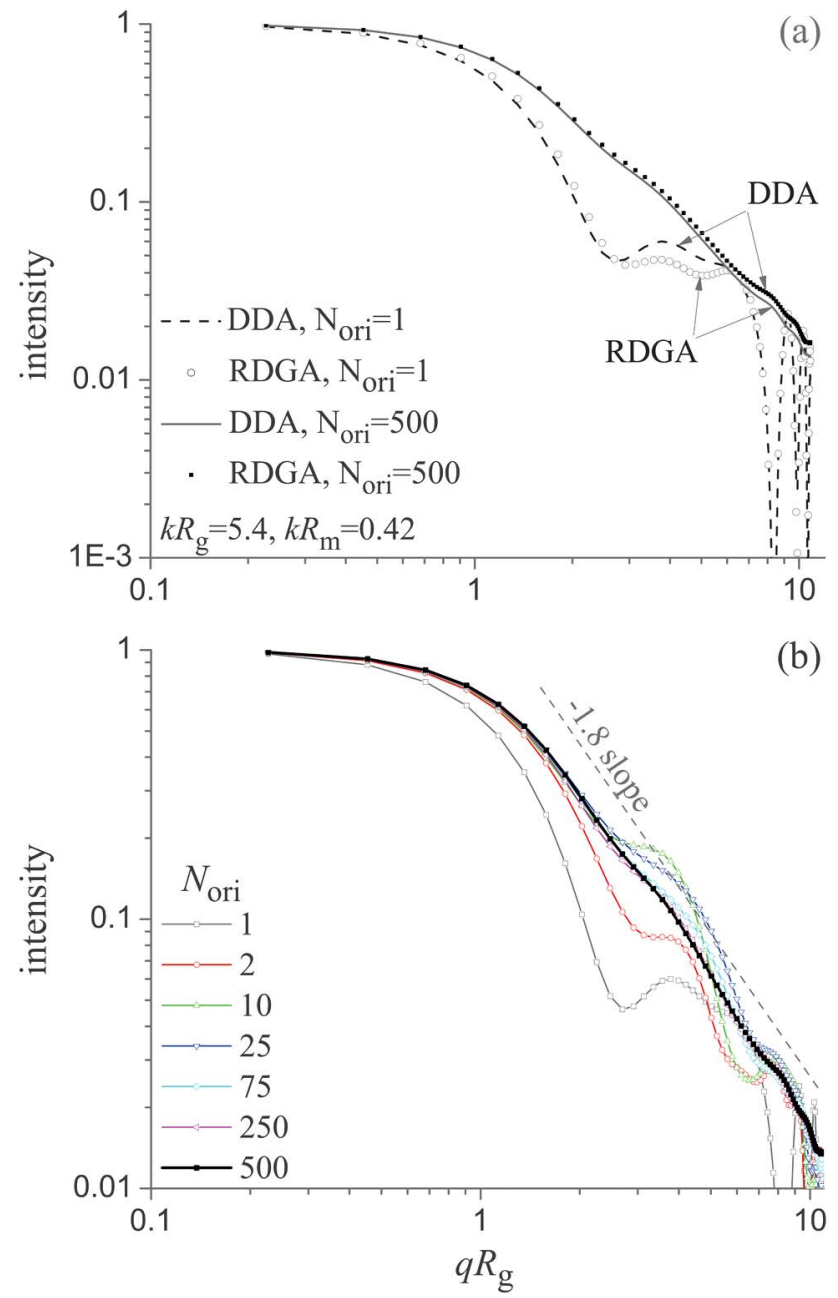

Fig. 3. Comparison between the DDA and RDGA scattered intensity. Both intensities are calculated using Eq. (6), except that the RDGA of the internal field is replaced by the incident field. Thus this calculation of the RDGA scattered intensity is essentially the square of the Fourier transform of the aggregate's spatial structure (see [22]). Plot (a) presents the comparison for an aggregate in a single orientation and averaged over $N_{\text {ori }}=500$ orientations. Plot (b) shows the DDA-calculated scattered intensity for a variety of aggregate orientations to demonstrate the effect that this averaging has to yield agreement with the power-law functionality of Eq. (2).

field magnitude tends to be slightly greater than the RDGA expectation.

The Stokes parameters $I, Q, U$, and $V$ are used to describe the polarization state of the far-field scattered wave. To render the state graphically, row (c) in Fig. 4 shows the unit sphere shaded to denote the wave's ellipticity along with the wave's vibration ellipse. The ellipticity is calculated from the Stokes parameters via Eq. (194) of [20], whereas the handedness, or rotation, of the polarization is indicated by the color: red denotes left-handed and blue denotes right-handed rotation. Inspection of the polarization state shows some degree of ellipticity for $N_{\text {ori }}=1$, including points in the horizontal scattering plane, which is wholly unexpected from the RDGA. In particular, the backscattering direction displays a nonzero ellipticity. This is consistent with the nonspherical shape of the aggregate in the context of the phasor cancellation model of the polarization state given in [23] and other studies 
(a)

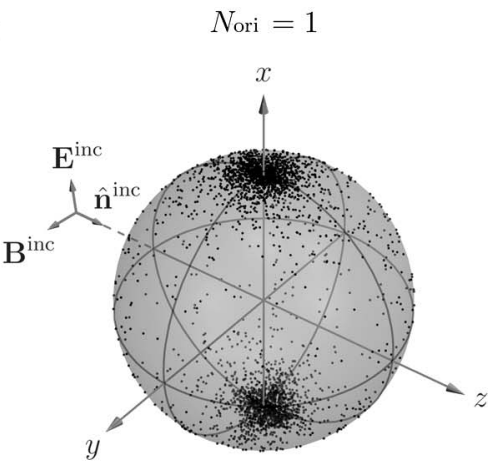

(b)

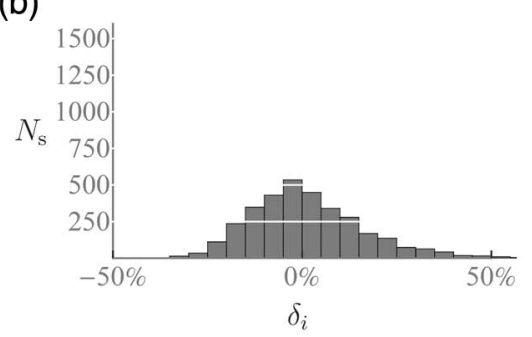

(c)

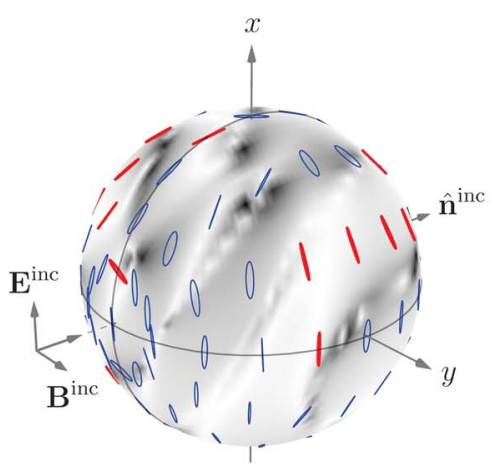

$N_{\text {ori }}=10$

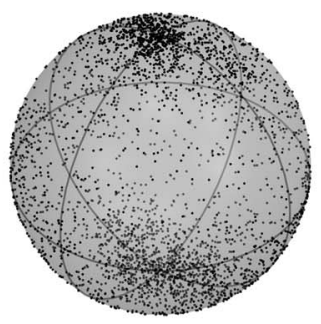

$$
N_{\text {ori }}=500
$$

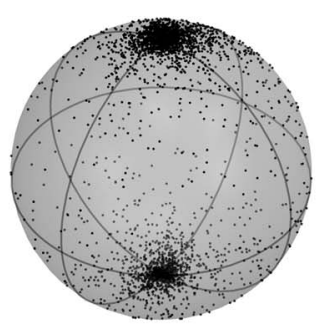

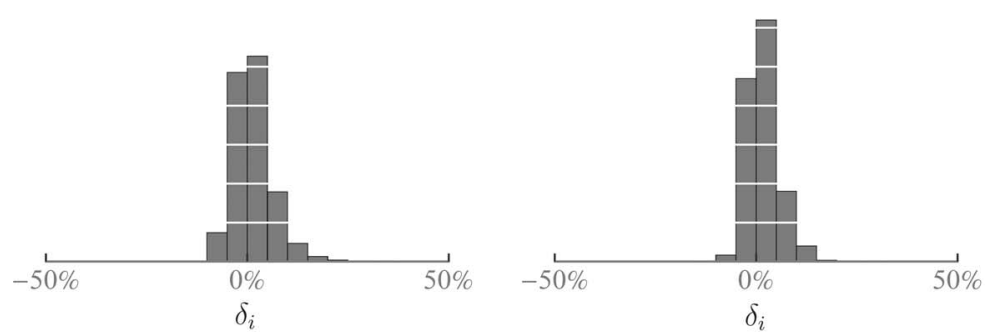
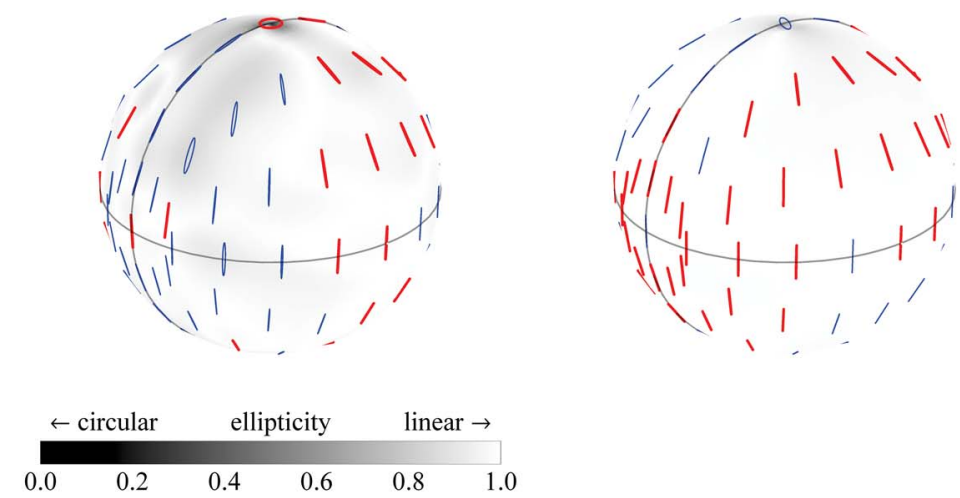

Fig. 4. Survey of the internal field and scattered wave's polarization state as a function of aggregate orientations. Row (a) shows the internal field directions given by Eq. (4), and row (b) shows histogram plots of the number of DDA lattice sites $N_{\mathrm{s}}$ with internal field deviation $\delta_{i}$ [Eq. (3)] in 5\% bins. Row (c) shows the polarization state of the far-field scattered wave across the unit sphere as calculated by the DDA via the Stokes parameters.

concerning the change in polarization state for nonspherical particles [20].

To say more, [24] shows that if the internal field is uniform, the scattered wave must be linearly polarized in all directions. If the RDGA were valid for an aggregate with spherical monomers, the polarization state would then be linear for all scattering angles. Indeed, this is the case in Fig. 4 once many orientations have been included. This implies that one reason why the polarization is linear is due to orientational averaging. Hence, a polarization measurement in the scattering plane for a single aggregate in a fixed orientation may show angular regions where the scattered wave is elliptical. Yet, the same measurement on a swarm of similar randomly oriented aggregates would show mostly linear polarization, which is consistent with laboratory observations [25].

As mentioned earlier, one can conclude from Figs. 2-4 that the RDGA is in error up to $\pm 25 \%$ in describing the aggregate's internal field, yet the far-field scattered intensity and polarization agrees well with the RDGA, especially when many orientations are considered. Thus there must be some special characteristic of the internal field that yields this agreement with the RDGA.

\section{ROLE OF FRACTAL GEOMETRY}

An explanation for the apparent validity of the RDGA relates to the fractal character of the aggregate. Given that $D_{\mathrm{f}}<2$, the aggregate has a nondense, porous structure [15]. Comparing this to a solid object $\left(D_{\mathrm{f}}=3\right)$ of similar refractive index, one could propose that the highly porous character of the aggregate reduces the electromagnetic coupling between monomers. Such a hypothesis could be tested quantitatively using the phase-shift parameter $\rho=2 k R|m-1|$, where $R$ is a suitable characteristic length. For a single spherical monomer, $\rho$ represents the difference in phase between a ray passing through the monomer diameter and a ray traversing the same distance in vacuum [26]. In other words, $\rho$ is a measure of the degree of refraction. Thus the two formal conditions for the validity of the RDGA mentioned in Section 3 can be combined into the single requirement that the phase-shift parameter for the aggregate be small. To account for the fractal geometry, 
the Maxwell-Garnet theory is used to define an effective refractive index (see [2]). With this, an effective phase-shift parameter for the aggregate would be

$$
\rho_{\text {eff }}=2 k|m-1| R_{\mathrm{m}}^{3-D_{\mathrm{f}}} R_{\mathrm{g}}^{D_{\mathrm{f}}-2} .
$$

Examination of Eq. (7) reveals that $\rho_{\text {eff }}$ decreases as the size of the aggregate increases provided that $D_{\mathrm{f}}<2$. Thus, in as much as Eq. (7) can describe the degree of refraction by an aggregate, it shows that the condition for the validity of the RDGA is met for sufficiently large aggregates. One should note that Eq. (7) is not the only way to understand the (unexpected) effectiveness of the RDGA (e.g., see [ㄴ,27,28]).
This discussion suggests that an evolution toward agreement with the RDGA will be seen for a $D_{\mathrm{f}}=1.8$ soot aggregate as its size increases. Such an evolution can be studied with the DDA; however, one cannot simply scale the size of the aggregate to vary $\rho_{\text {eff }}$. In order to keep the morphologic and electromagnetic properties of the aggregate constant as much as possible, the aggregate size is varied through the number of monomers $N_{\mathrm{m}}$, with $R_{\mathrm{m}}$ and $m$ fixed. This is done using a parent-child model: the aggregate in Figs. 1-4 above, which will be called the parent, is truncated by a sphere of radius $R_{\mathrm{t}}$ positioned at the center of mass. All monomers outside of this sphere are discarded, and what remains becomes the child aggregate. In this way, aggregates of varying size

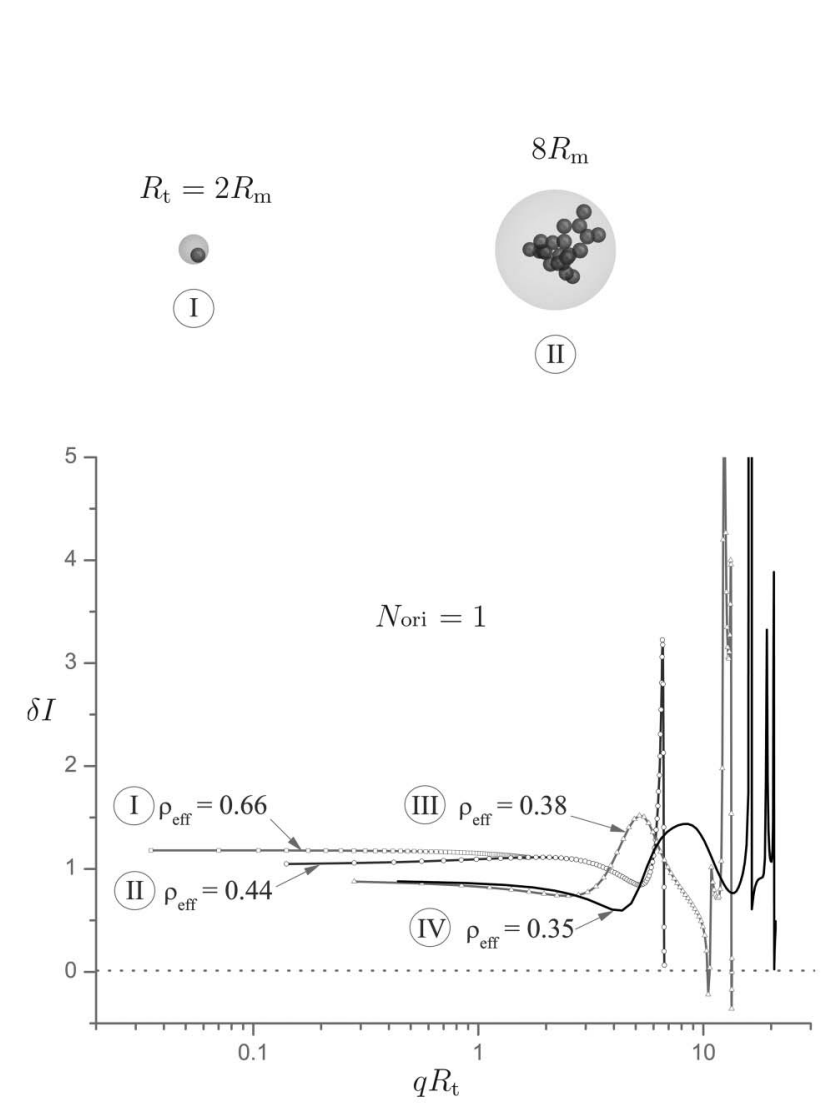

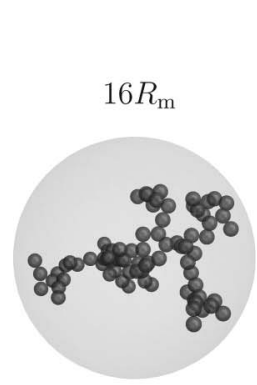

(III)

$$
25 R_{\mathrm{m}} \sim 2 R_{\mathrm{g}}
$$

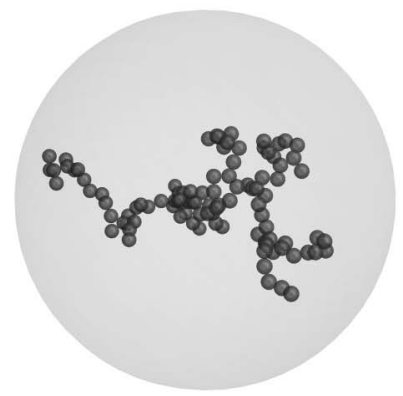

IV
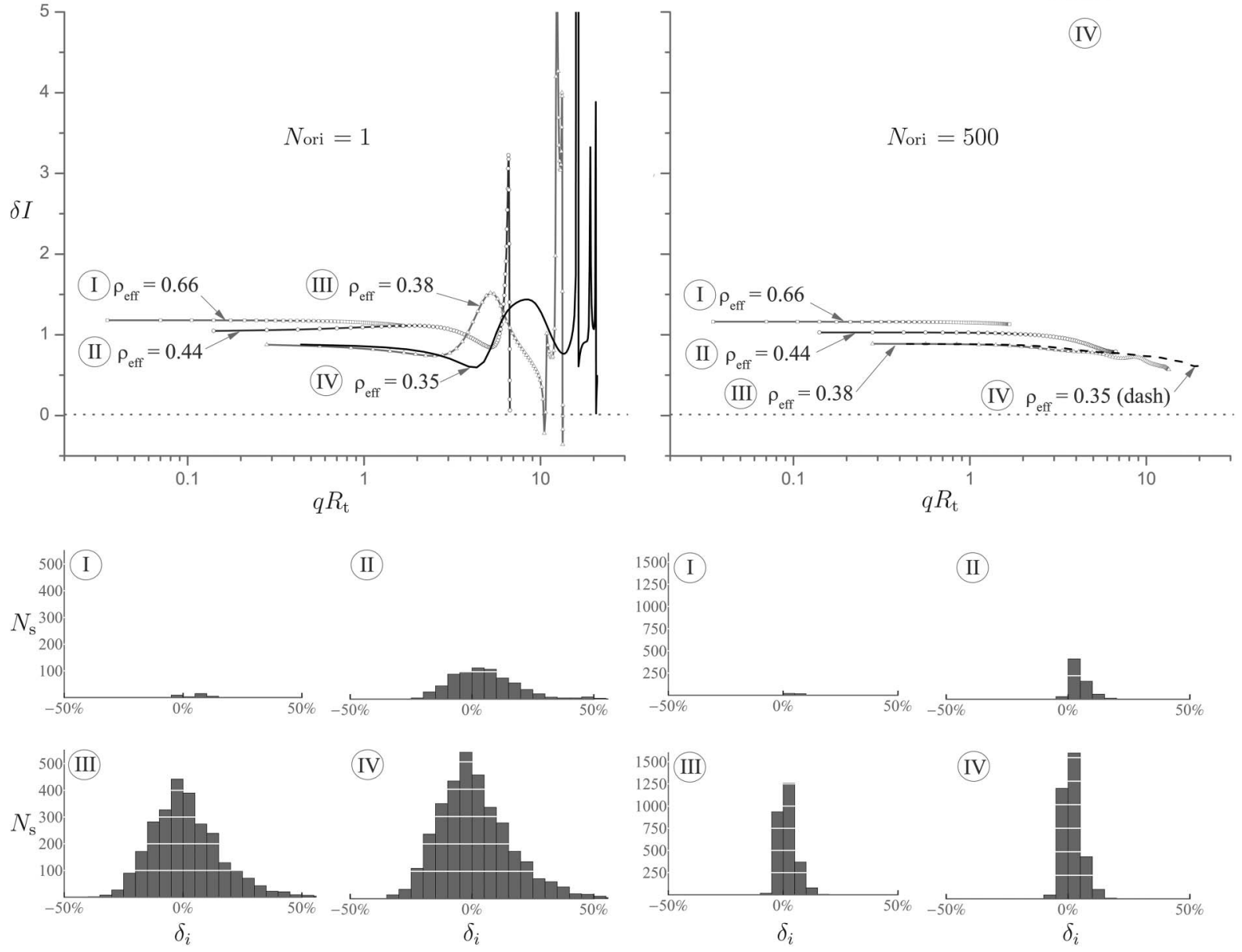

Fig. 5. Comparison of the DDA and RDGA scattered intensity and internal field as a function of increasing aggregate size. The parent aggregate is shown in IV and is the same aggregate used in Figs. $1-4$. Smaller child aggregates are generated from this parent by truncating the parent with a sphere of radius $R_{\mathrm{t}}$. The middle plots show the percent deviation $\delta I$ [Eq. (8)] of DDA and RDGA scattered intensity for aggregates in a single orientation and an ensemble of 500 orientations. The bottom plots show the number of lattice sites $N_{\mathrm{s}}$ with internal field magnitude deviations $\delta_{i}[$ Eq. (3)] in $5 \%$ bins. 
can be made that have some structural similarity with each other. Ideally, this structural similarity would mean that the fractal dimensions of the child and parent are equivalent. However, the range of $R_{\mathrm{t}}$ considered is such that the smallest child aggregate consists of a single monomer only. In that case, the fractal dimension is $D_{\mathrm{f}}=3.0$, whereas the largest aggregate, i.e., the parent, has $D_{\mathrm{f}}=1.8$. Note that in some sense, this procedure is similar to the evolving aggregate-size structure factor measurements in [16].

Figure 5, top row, shows the parent and three child aggregates each labeled by Roman numerals. Also shown are the truncation spheres as measured in terms of $R_{\mathrm{m}}$. To test the predictive ability of Eq. (7), the scattered intensity is calculated for each aggregate using the DDA and RDGA, i.e., $I_{\text {dda }}$ and $I_{\text {rdga }}$, respectively. The relative deviation as a function of $q R_{\mathrm{t}}$ between these is then calculated as

$$
\delta I\left(q R_{\mathrm{t}}\right)=\frac{I_{\mathrm{dda}}\left(q R_{\mathrm{t}}\right)-I_{\mathrm{rdga}}\left(q R_{\mathrm{t}}\right)}{I_{\mathrm{rdga}}\left(q R_{\mathrm{t}}\right)} .
$$

If $\delta I=0$, then the DDA and RDGA yield equivalent scattered intensity, whereas a nonzero value indicates percent deviation between the two.

The middle panel in Fig. 5 shows $\delta I$ for the aggregates in a single orientation and averaged over $N_{\text {ori }}=500$ orientations. The values of $\rho_{\text {eff }}$ are also shown. For both single and random orientations, larger aggregates show less overall deviation from the RDGA, in agreement with Eq. (7). Interestingly, the average deviation $\delta I$ is roughly the same for aggregates of the same size, regardless of whether or not they are orientationally averaged. The primary effect of the averaging is to wash out the spikes in deviation that are seen in the large $q R_{t}$ range for aggregates in fixed orientations. The bottom panels in Fig. 5 show histogram plots of the number of lattice sites $N_{\mathrm{s}}$ in each aggregate with internal field magnitude deviation $\delta_{i}$ [Eq. (3)] in bins of 5\%. Here, one can see that the distributions grow in amplitude with aggregate size, which is simply due to the larger number of sites contained within aggregates of increasing size. The important behavior is contained in the distribution shape, in particular its width. For aggregates in a single orientation, the deviation is large, up to $\pm 50 \%$, whereas this is reduced to $\pm 25 \%$ for orientationally averaged aggregates, consistent with Fig. 4 . However, the shape of the distributions is roughly symmetric about $\delta_{i}=0$, meaning that on average there are as many lattice sites with internal field magnitude greater than the RDGA prediction as there are with magnitude less than the RDGA.

Formally, the internal field is connected to the scattered intensity by the VIE, which integrates the internal field throughout the aggregate [recall Eq. (6)]. Thus the mostly symmetric shape of the field-deviation distributions in Fig. $\underline{5}$ suggests that this integration averages out the positive and negative deviations, yielding the unexpectedly good agreement with the RDGA for $I$. The slight asymmetry of the distributions may then account for the small error ultimately observed in the RDGA. Had the phase-shift parameter of the aggregate been calculated with the monomer refractive index and radius of gyration, one would find that increasing aggregate size would give an increasing phase shift, and thus no enhanced agreement with the RDGA would be expected. By building in the fractal nature of the aggregate using the
Maxwell-Garnet theory, the phase shift of Eq. (7) decreases with increasing aggregate size and thus correctly predicts the improved agreement with the RDGA.

\section{VALIDITY OF THE MODEL}

There is some question how valid the DDA may be when applied to the high refractive index and complex shape of the fractal aggregates considered here. For example, a study by $[29,30]$ using the T-matrix method to model scattering from aggregates of interacting spheres finds slow convergence of the number of terms in the harmonic expansions involved. Even though the spherical monomers are Raleigh-type particles, due to the strong interactions between them [29] found that up to 10 terms may be required in the expansion for a bisphere aggregate for acceptable convergence to be obtained. Such results could suggest that the DDA cannot be used to model scattering from fractal aggregates as is done here. To investigate this question, Fig. $\underline{6}$ shows the effect on $I_{\mathrm{dda}}$ as the number of dipoles per monomer $N_{\mathrm{dda}} / N_{\mathrm{m}}$ is increased using the following values $N_{\mathrm{dda}} / N_{\mathrm{m}}=4,8,14,23,34,48,65$, 88 , and 113 . This ratio is the primary quantity controlling the

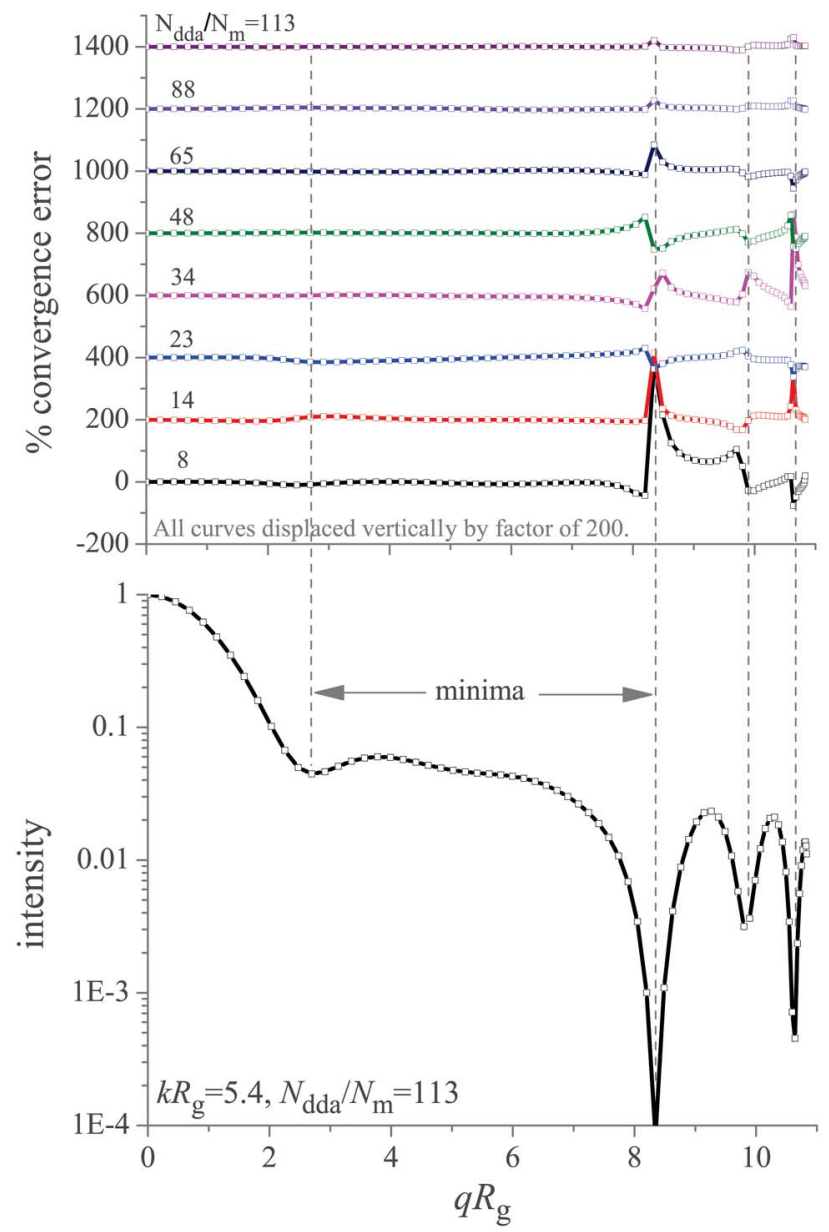

Fig. 6. Relative error between DDA scattered intensity curves using successively greater numbers of dipoles per monomer $N_{\mathrm{dda}} / N_{\mathrm{m}}$. The upper plot shows the convergence error as described by Eq. (9) where the number of dipoles per monomer is indicated on each curve. The aggregate is the parent aggregate of Fig. 5. The lower plot shows the scattered intensity curve for the largest, i.e., most accurate, value for $N_{\text {dda }} / N_{\mathrm{m}}$. Here, one can see the correlation between the minima in the scattered intensity and the spikes in the convergence error above. 
accuracy of the calculated scattered intensity. Using the parent aggregate in Fig. 5, i.e., the largest aggregate, the relative error between $I_{\mathrm{dda}}$ as calculated by using an increasing number of dipoles is shown in the upper plot. This is called the "convergence error" here. For example, the bottom curve in the error plot is calculated in analogy to Eq. () as

$$
\frac{I_{\mathrm{dda}}^{(8)}\left(q R_{\mathrm{t}}\right)-I_{\mathrm{dda}}^{(4)}\left(q R_{\mathrm{t}}\right)}{I_{\mathrm{dda}}^{(8)}\left(q R_{\mathrm{t}}\right)} \times 100,
$$

where $I_{\text {dda }}^{(4)}$ means that the DDA scattered intensity is calculated using $N_{\mathrm{dda}} / N_{\mathrm{m}}=8$ dipoles per monomer and is compared to the same calculation using $N_{\mathrm{dda}} / N_{\mathrm{m}}=4$. On average, the error is less than $10 \%$ when $N_{\mathrm{dda}} / N_{\mathrm{m}}=34$ or larger values are used. The exception is in the neighborhood of minima in the scattered intensity curves, where numerical errors resulting from the subtraction of two small numbers cause spikes in the curves. The figure shows a plot of the scattered intensity using the largest dipole ratio $N_{\mathrm{dda}} / N_{\mathrm{m}}=$ 113 where the minima in the curve are correlated with the spikes in the error. What Fig. 6 shows is that increasing the number of dipoles per monomer produces a scattering curve that converges to a "master curve" as more dipoles are included, i.e., a curve approximately given by the $N_{\mathrm{dda}} / N_{\mathrm{m}}=$ 113 case. In this context, the value of $N_{\mathrm{dda}} / N_{\mathrm{m}}=34$ that is used above would seem to be acceptable for relative errors less than $10 \%$. It is plausible, then, that this assessment of accuracy also extends to the internal fields provided by the same DDA calculations. Such validity is an important assumption in this work, and there would be value to further investigating this issue. For example, the same superposition T-matrix theory used in [30] could be applied to the aggregates considered here, which would provide the analytically exact scattered intensity. However, what is really needed is a retooling of the simulation code in [29,30] for the intermal fields; those could then be directly compared to the DDA fields calculated here. Unfortunately, such comparison is not possible within the scope of this work.

\section{CONCLUSION}

Using the DDA, this work shows that the internal electric field within a simulated soot fractal aggregate can deviate up to $\pm 50 \%$ from the RDGA prediction, yet the DDA far-field scattered intensity agrees well with this prediction. The validity of the approximation for the scattered intensity, despite its poor description of the internal field, relates to the manner in which the true field deviates from that assumed in RDGA. The DDA shows that roughly equal portions of the aggregate exhibit internal field magnitudes greater than, and less than, the RDGA value. Consequently, when the internal field is integrated by the VIE to yield the scattered intensity, the average result is well described by the RDGA. This behavior is seen for aggregates in both fixed and random orientations; in the latter case, the classic $Q$-space power-law functionality is seen.

\section{ACKNOWLEDGMENTS}

The authors are grateful for the helpful comments of two anonymous reviewers and discussions with Nikolai Khlebtsov, Roamin Ceolato, and Nicolas Riviere. This work was partly supported by the U.S. Army Research Office.

\section{REFERENCES}

1. T. C. Bond and R. W. Bergstrom, "Light absorption by carbonaceous particles: an investigative review," Aerosol Sci. Technol. 40, 27-67 (2006)

2. C. M. Sorensen, "Light scattering by fractal aggregates: a review," Aerosol Sci. Technol. 35, 648-687 (2001)

3. L. Liu and M. I. Mishchenko, "Effects of aggregation on scattering and radiative properties of soot aerosols," J. Geophys. Res. 110 D 11211 (2005).

4. M. V. Berry and I. C. Percival, "Optics of fractal clusters such as smoke," Opt. Acta 33, 577-591 (1986).

5. T. L. Farias, Ü. Ö. Köylü, and M. G. Carvalho, "Range of validity of the Rayleigh-Debye-Gans theory for optics of fractal aggregates," Appl. Opt. 35, 6560-6567 (1996).

6. G. Wang and C. M. Sorensen, "Experimental test of the Rayleigh-Debye-Gans theory for light scattering by fractal aggregates," Appl. Opt. 41, 4645-4651 (2002).

7. H. Y. Chen, M. F. Iskander, and J. E. Penner, "Light scattering and absorption by fractal agglomerates and coagulations of smoke," J. Mod. Opt. 37, 171-181 (1990).

8. Y. Zhao and M. Lin, "Assessment of two fractal scattering models for the prediction of the optical characteristics of soot aggregates," J. Quant. Spectrosc. Radiat. Transfer 110, 315-322 (2009).

9. R. Dhaubhadel, C. S. Gerving, A. Chakrabarti, and C. M. Sorensen, "Aerosol gelation: synthesis of a novel, lightweight, high specific surface area material," Aerosol Sci. Technol. 41, 804-810 (2007).

10. F. G. Pierce, "Aggregation in colloids and aerosols," Ph.D. dissertation (Kansas State University, Manhattan, Kansas, 2007).

11. C. M. Sorensen and G. C. Roberts, "The prefactor of fractal aggregates," J. Colloid Interface Sci. 186, 447-452 (1997).

12. S. P. Kearney and F. Pierce, "Evidence of soot superaggregates in a turbulent pool fire," Combust. Flame 159, 3191-3198 (2012).

13. C. M. Sorensen, W. Kim, D. Fry, D. Shi, and A. Chakrabarti, "Observations of soot superaggregates with a fractal dimension of 2.6 in laminar acetylene/air diffusion flames," Langmuir 19, 7560-7563 (2003).

14. R. Dhaubhadel, F. Pierce, A. Chakrabarti, and C. M. Sorensen, "Hybrid superaggregate morphology as a result of aggregation in a cluster-dense aerosol," Phys. Rev. E 73, 011404 (2006).

15. A. M. Brasil, T. L. Farias, M. G. Carvalho, and U. O. Koylu, "Numerical characterization of the morphology of aggregated particles," Aerosol Sci. Technol. 32, 489-508 (2001).

16. H. X. Zhang, C. M. Sorensen, E. R. Ramer, B. J. Olivier, and J. F. Merklin, "In situ optical structure factor measurement of an aggregating soot aerosol," Langmuir 4, 867-871 (1988).

17. C. M. Sorensen, J. Cai, and N. Lu, "Light-scattering measurements of monomer size, monomers per aggregate, and fractal dimension for soot aggregates in flames," Appl. Opt. 31, 6547-6557 (1992).

18. K. C. Smyth and C. R. Shaddix, "The elusive history of $m=1.57-0.56 \mathrm{i}$ for the refractive index of soot," Combust. Flame 107, 314-320 (1996).

19. C. F. Bohren and D. R. Huffman, Absorption and Scattering of Light by Small Particles (Wiley, 1998).

20. M. I. Mishchenko, L. D. Travis, and A. A. Lacis, Scattering, Absorption, and Emission of Light by Small Particles (Cambridge University, 2002).

21. M. A. Yurkin and A. G. Hoekstra, "The discrete dipole approximation: an overview and recent developments," J. Quant. Spectrosc. Radiat. Transfer 106, 558-589 (2007).

22. M. J. Berg, "Power-law patterns in electromagnetic scattering: a selected review and recent progress," J. Quant. Spectrosc. Radiat. Transfer 113, 2292-2309 (2012)

23. M. J. Berg, C. M. Sorensen, and A. Chakrabarti, "Reflection symmetry of a sphere's internal field and its consequences on scattering: a microphysical approach," J. Opt. Soc. Am. A 25, 98-107 (2008).

24. M. J. Berg, "Reflection symmetry of a sphere's internal field and its consequences on scattering: behavior of the Stokes parameters," in Polarimetric Detection, Characterization and Remote Sensing, M. I. Mishchenko, Y. S. Yatskiv, V. K. Rosenbush, and 
G. Videen, eds., NATO Science for Peace and Security Series C: Environmental Security (Springer, 2011), pp. 31-48.

25. N. Lu and C. M. Sorensen, "Depolarized light scattering from fractal soot aggregates," Phys. Rev. E 50, 3109 (1994).

26. M. Kerker, The Scattering of Light and Other Electromagnetic Radiation (Academic, 1969).

27. N. G. Khlebtsov, "Optics of fractal clusters in the anomalous diffraction approximation," J. Mod. Opt. 40, 2221-2235 (1993).
28. N. G. Khlebtsov and A. G. Melnikov, "Structure factor and exponent of scattering by polydisperse fractal colloidal aggregates,” J. Colloid Interface Sci. 163, 145-151 (1994).

29. M. I. Mishchenko, D. M. Mackowski, and L. D. Travis, "Scattering of light by bi-spheres with touching and separated components," Appl. Opt. 34, 4589-4599 (1995).

30. D. M. Mackowski and M. I. Mishchenko, "Calculation of the T matrix and the scattering matrix for ensembles of spheres," J. Opt. Soc. Am. A 13, 2266-2278 (1996). 